\title{
Integrating BIMserver and OpenStudio for Energy Efficient Building
}

\author{
Nan $\mathrm{Yu}^{1}$, Yufei Jiang ${ }^{1}$, Lannan Luo ${ }^{1}$, Sanghoon Lee ${ }^{2}$, Abdou Jallow ${ }^{2}$, Dinghao Wu ${ }^{1}$, \\ John I. Messner ${ }^{2}$, Robert M. Leicht ${ }^{2}$, and John Yen ${ }^{1}$ \\ ${ }^{1}$ College of Information Sciences and Technology, The Pennsylvania State University, \\ University Park, PA 16802. Email: \{nuy106, yzj107, lzl144, dwu, jyen\}@ist.psu.edu \\ ${ }^{2}$ Department of Architectural Engineering, The Pennsylvania State University, \\ University Park, PA 16802. Email: \{Sanghoon.Lee, ajallow, jmessner, \\ rmleicht\}@engr.psu.edu
}

\begin{abstract}
Energy Efficient Building (EEB) design requires many simulation tools to support making decisions for optimized building solutions, resulting in frequent interactions between computational tools. Building Information Modeling (BIM) server platforms can support the storage, maintenance, and query of IFC-based building information models. However, the lack of a unified interface to support information exchange and interoperability among different building design and simulation tools has become a bottleneck of the EEB design process. This paper describes an integrated approach at the data level to combining BIMserver and OpenStudio to build a unified EEB data exchange model. Our first step is to build an information exchange bridge between BIMserver and OpenStudio, which will enable different design and simulation tools that are connected to either of them to interoperate and exchange needed data. In the paper, we also discuss the challenges of the seamless integration due to the dependency on both BIMserver and OpenStudio. The integrated approach, which organizes the data flow in a unified model, enabling effective exchange of data, is currently in a beta-testing phase.
\end{abstract}

Keywords: BIMserver, OpenStudio, Energy Efficient Building (EEB), Data Exchange Model

\section{INTRODUCTION}

Energy consumption has gained much attention recently. In the Architecture, Engineering, Construction and Facilities Management (AEC/FM) field, energy efficient building design is becoming more critical, especially as it relates to energy retrofit projects. In the process of energy efficient building design, decision-making in the very early stages might significantly influence the energy consumption (Pollock et al., 2009). The decision-making process should be built upon a channel, which connects the computational representation of a building's energy elements and the corresponding economic considerations (Jones et al., 2010). Energy modeling is such a channel providing designers with an outlook of potential energy consumption of varieties of designs prior to constructing the building (Fleming et al., 2012).

During the building design lifecycle, Energy Efficient Building (EEB) design depends on the collaboration project participants using a variety of simulation tools to 
make decisions for the optimized building solutions. Currently, different simulation tools running in different "energy simulation views" (Bazjanac, 2008) determine the varieties of data sets and data formats (Bazjanac \& Kiviniemi, 2007). It is necessary to gather all simulation views into an integrated whole-building simulation methodology with the intent of exchanging data seamlessly (Guglielmetti et al., 2011).

Some Building Information Modeling (BIM) server platforms, such as 'BIMserver' implemented by bimserver.org (Beetz el al., 2010) support the storage, maintenance, and query of Industry Foundation Classes (IFC) based building information models. OpenStudio, an interface to support whole building energyrelated modeling and simulations, has another set of building energy modeling (BEM) representations (Weaver et al., 2012). A disconnect between different models prevents architects, engineers, and researchers from easily conducting integrated whole-building energy analysis (Guglielmetti et al., 2011). Accordingly, this paper explores an integrated approach to leverage BIMserver and OpenStudio to enable open data exchange and interoperability among different building design and simulation tools. With the integrated approach, the inherent data inconsistency and mapping problems could also be solved.

\section{BACKGROUND}

BIM for energy efficient building design. The AEC/FM industry shows increasing interest in adopting information technology in building designs (Bazjanac \& Kiviniemi, 2007). BIM acts as a bridge between the industry and information technology (Eastman et al., 2008), which makes the entire building lifecycle more efficient and effective. BIM, an interoperable data model, can enable bi-directional data service for various simulation tools in the AEC/FM industry projects, which means the import and export of relevant data must be compatible with other tools (Bazjanac, 2007; O’Donnell et al., 2011).

Current simulation tools for building energy analysis. Building energy performance simulation leverages computer-based building energy analysis to quantitatively validate the correctness of decisions on building design and operations (Bazjanac et al., 2011). High-performance buildings require an integrated analysis, including whole-building energy, daylighting, airflow, among others. Traditionally, different simulation tools focus on their own domains. For instance, CONTAM is a multi-zone airflow and contaminant transport analysis software (Walton \& Dols, 2010), EnergyPlus performs whole-building energy analysis, and Radiance is used for daylight and electric lighting simulation (Guglielmetti \& Scheib, 2012). Typically, designers leveraging these tools use a point-to-point data exchange model (O’Donnell et al., 2011), which is often very complicated and inefficient. In order to facilitate information flow from an architectural design model to an energy model, Bazjanac and Kiviniemi (2007) developed a tool called the Geometry Simplification Tool (GST) that simplifies valid IFC geometric model and extract construction properties.

Building information representations. One of the essential barriers preventing seamless data exchange is the different building information representations. Each representation offers a range of proprietary file formats used in 
modeling and simulation tools; EnergyPlus input data file (IDF), Green Building XML (gbXML) and DXF are some of the most common data representations and input file formats used in energy-related simulation tools. On the other hand, Industry Foundation Classes (IFC) is the most mature open standard to represent building information and some BIM tools including the BIMserver support the storage, maintenance and query of the IFC-based BIM models. Due to the difference between the information representations, the transformation among them introduces human intervention, which also makes the process inefficient and error prone. Therefore, a highly integrated and easily shared information exchange model is required.

\section{THE INTEGRATED DATA EXCHANGE MODEL}

The commercially available BIM authoring applications do not include the HVAC system or operation schedules within the IFC export. Therefore, a link between building information exported in IFC format from BIMserver and BEM is required for an integrated data exchange model to support the whole simulation process.

Some initial work to investigate information exchange between BIM tools and simulation tools was performed using airflow analysis and to develop the integrated data exchange model to support the interoperability. Figure 1 shows an airflow analysis model development workflow using BIM (Jiang et al., 2013). The horizontal level stands for the simulation process inside CONTAM, including zoning, specifying flow path, connecting HVAC to zones and running the simulation. It is of critical importance that we build a tool to collect building information specified by the requirements and connect CONTAM with BIMserver. During the information collection process, a subset of building geometric information would be extracted via a query function according to MVD (model-view-definitions). The output of the tool is a subset of input data that CONTAM understands.

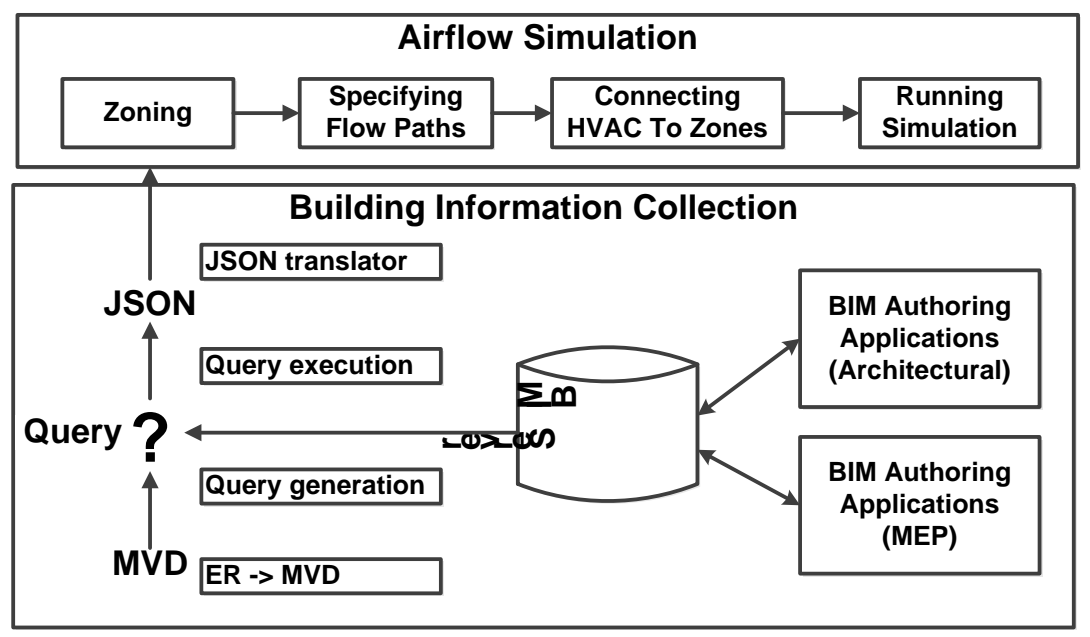

Figure 1. A Representative Workflow: Airflow Analysis Model Development Using BIM 


\section{Integration through OpenStudio}

The workflow in Figure 1 provides a feasible method for airflow analysis through direct information extraction from an IFC model into CONTAM. However, as mentioned in the background section, building information has various representations and building energy modeling usually works in different "energy simulation views". A point-to-point communication channel needs to be established for each direct data exchange. The complexity of the point-to-point information exchange model would increase dramatically as the number of simulation tools increases. Thus, the AEC/FM industry experts and IT professionals are pursuing integrated models to eliminate the need for distinct data models and management services at the individual tool level. Jiang et al. (2012) propose that the integration of BIMserver and OpenStudio might be a potential solution for information exchanges in the energy simulation domain. Based on this assumption, we have analyzed the feasibility to choose OpenStudio as a middleware interface.

OpenStudio is designed to establish an object-oriented framework for Building Energy Models (BEM), which is compatible with existing work (Guglielmetti, 2011). BIMserver is an object-oriented framework for building information and transforms all semi-structured information presented in IFC into building objects with corresponding attributes and relationships stored in memory. In view of the object-oriented characteristic, it is possible for data to flow from the BIMserver to OpenStudio and build an information exchange bridge for building simulations. Second, OpenStudio and EnergyPlus are bound together in the initial design (Ellis et al., 2008). Accordingly, EnergyPlus leverages OpenStudio to conduct whole-building energy analysis without extra effort. OpenStudio also supports Radiance to perform daylighting analysis besides conducting whole building energy analysis through EnergyPlus. It is desirable to adopt a single tool to facilitate analyses from different perspectives.

OpenStudio is designed to overcome the shortage of user-friendliness of EnergyPlus and Radiance. Lack of an intuitive graphical user interface (GUI) for the tools creates a high entry barrier which prevents novel users from using the tools (Weaver et al., 2012). Other software applications such as DesignBuilder, IES, and eQuest try to present a state-of-the-art GUI to users. However, some of these software applications are commercial software. Users are constrained by the GUI provided to make limited analyses. In contrast, OpenStudio is open-source, cross-platform and cross-language. Last but not least, OpenStudio provides a rapid development mode and open application programming interface (API), which makes it highly extensible and customizable. It is rather simple for developers to either build on existing applications or create completely new ones to conduct customized building energy analysis (Weaver et al., 2012). All of these aspects suggest OpenStudio as a suitable platform for initial targeting to support the data exchange needs of building energy modeling. 


\section{The proposed integrated data exchange model}

Figure 2 shows the proposed framework of integrating BIMserver and OpenStudio to build an integrated EEB data exchange model. The first step is to build an information exchange bridge between BIMserver and OpenStudio, which enables different design and simulation tools to connect to either of them to interoperate and exchange needed data.

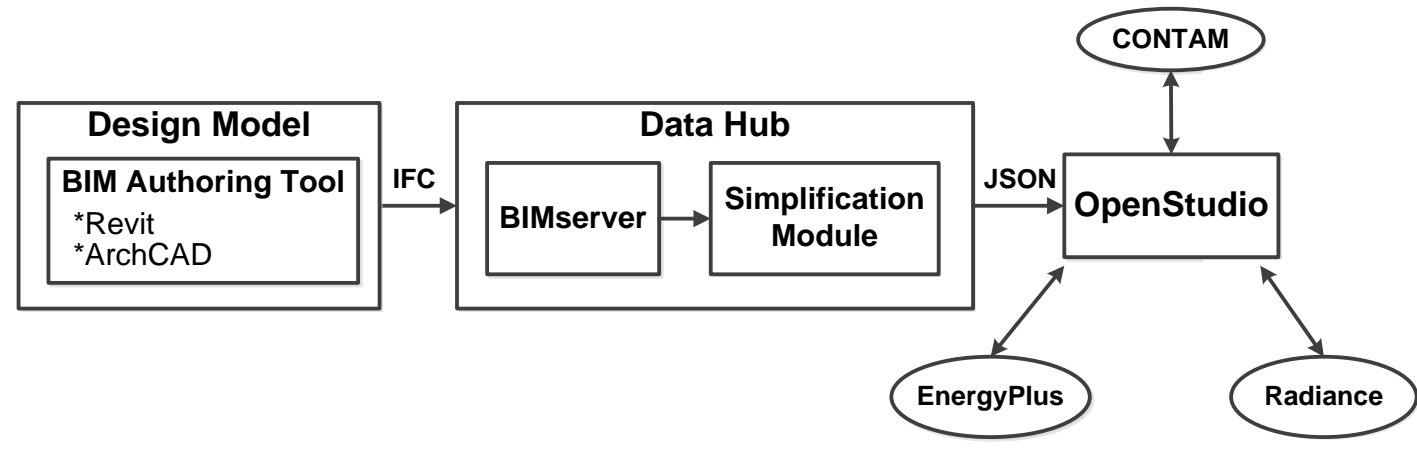

Figure 2. The Proposed Integrated EEB Data Exchange Model

In the model, BIMserver is adopted as the implementation of a BIM server which takes charge of building information management and selective building information retrieval. It accepts IFC format exported from BIM authoring tools such as Autodesk ${ }^{\circledR}$ Revit ${ }^{\circledR}$ or other tools that support IFC exporting, as input data. The input IFC data contains overall and original building geometric information and is loaded into the memory of BIMserver.

However, OpenStudio requires a reduced and simplified data set instead of the complex and rich BIM data. Therefore, the exchange needs to extract the simplified geometry from memory and transmit it to OpenStudio, so a simplification module is shown inside the model. It automatically generates Java query code according to IFC specification. The Java code is inserted and run in the advanced query area in the GUI of BIMserver, which searches the memory and displays the query result in the console. The simplification module not only provides data set reduction and simplification, but also offers the function of data translation and data interpretation, which transforms the retrieved information into the appropriate data set and format to keep the inconsistency in different views. For example, most elements in a building are described under different local coordination systems, but the resulting set is required in the global coordination system. Therefore, the translation between various local coordination systems is part of the simplification module. The translation leverages the matrix transformation to relocate the origin and the directions of the axes. Furthermore, IFC specification is illustrated in an inheritance graph, where the bottom entities inherit the attributes and relationships from the top entities. In that case, the attributes and relationships of the bottom entities are interpreted by the derived data. Through reduction and simplification, data translation and interpretation, 
original data is transformed into the required data set available under different views. The above steps are to prepare the data structure.

Another function of the simplification module is data transformation, including the relationship mapping and format transformation. Data that flows into BIMserver is in IFC format, while the output format is supposed to be OpenStudio Model (OSM). These two different formats contain different sets of data properties. The mapping process from IFC to OSM is based on the properties analysis in different views. The format transformation is accomplished via JSON format. JSON (JavaScript Object Notation) is a lightweight open data-interchange format (http://www.json.org). All the data which passes through BIMserver is objectoriented so that it can organize the data as building objects and export the query result in JSON format. The data in the JSON format needs to be parsed and re-organized into OSM format, which is the required and accepted input format by OpenStudio. This can be implemented by a programming script in either Ruby or C\#.

With the link between BIMserver and OpenStudio, the integrated model enables data exchange and the pipeline of whole-building energy. The pipeline refers to a chain of building information processing stages. For example, data processed in a design model is exported into the IFC format and taken by BIMserver, which selectively transforms the needed data for OpenStudio. Other tools linked with OpenStudio can run whole-building energy simulations to conduct energy analysis. In the data transformation from BIMserver to OpenStudio, the middleware interface can extract geometric points of building elements and simplified elements, such as walls with regular shapes. We also analyzed the concrete building examples in both IFC and OSM in expectation of creating transformation rules from IFC to OSM. With the integrated data exchange model, we are able to pipeline the data flow in a unified interface and thus enable effective exchange of data.

\section{Challenges}

Integrating simplification module into BIMserver. Currently the simplification module and BIMserver run in their own isolated workspaces. Users still need to paste the automatically generated Java query code from the simplification module into the GUI provided by BIMserver and then copy the query result back to the simplification module for exporting. It would be more efficient to eliminate human intervention completely by integrating the simplification module into BIMserver as a plug-in. BIMserver is an open-source project that allows modifying the source code and adding customized modules to serve for the integrated data exchange model.

Seamless information exchanges from simplification module to OpenStudio. The integration of the BIMserver and OpenStudio has a dependency on the JSON format which bridges the gap between IFC and OSM formats. Presently, OpenStudio does not support parsing JSON format directly but offers an open rapid development mode and API. As a result, the JSON format produced by the simplification module will rely on a script to transform JSON into OSM, which adds a new dependency on an extra external module. It is suggested that such a function should be embedded inside the framework of OpenStudio. 


\section{CONCLUSION}

A high-performance building requires comprehensive whole-building energy analyses to optimize energy consumption. AEC/FM industry experts should leverage information technology and various simulation tools to conduct integrated analyses to assist with their decision-making in the whole building lifecycle. This paper proposes the concept of the unified EEB data exchange approach that integrates BIMserver and OpenStudio, and analyzes the feasibility of an integrated model. In the EEB data exchange model, BIMserver performs the information management and selective building geometry retrieval, and OpenStudio plays the role of middleware connecting other simulation tools. The unified model shows the potential of supporting domain experts to easily conduct various energy-related analyses without reproducing the same building information. The next step is to complete the beta test of the data exchange model. The prospect of the integrated model also drives us to overcome the challenges above and set up an enhanced EEB data hub encompassing both BIMserver and OpenStudio to achieve seamless data exchange.

\section{ACKNOWLEDGEMENT}

This research is supported in part by Department of Energy (DOE) under the Energy Efficient Buildings Hub (EEB Hub) project (http://www.eebhub.org/).

\section{REFERENCES}

Bazjanac, V. \& Kiviniemi, A. (2007). "Reduction, simplification, translation and interpretation in the exchange of model data." In Proceedings of the 24th Conference. Bringing ITC knowledge to work: 163-168.

Bazjanac, V. (2008). "IFC BIM-based methodology for semiautomated building energy performance simulation." In Proceedings of the 25th International Conference on Information Technology in Construction, Santiago, CL: 292299.

Bazjanac, V., Maile, T., O’Donnell, J., Rose, C., Mrazovic, N., (2011) "Data Enviroments and Processing in SemAutomated Simulation with EnergyPlus." In: CIB W078-W102: 28th International Conference. CIB, Sophia Antipolis, France.

Beetz, J., Berlo, L.-V., Laat, R.-d. and Helm, P.-v., (2010). "Bimserver.org - An Open Source IFC Model Server". In Proceedings of the CIP W78 conference, Cairo.

Eastman, C., Teicholz, P., Sacks, R. and Liston, K., (2008). "BIM Handbook", Wiley \& Sons.

Ellis, P.G., Torcellini, P.A., Crawley, D.B.. (2008). "Energy Design Plug-in: An EnergyPlus Plug-in for SketchUp." In Proceedings of the IBPSA-USA SimBuild Conference, Berkeley, California.

Fleming, K., Long, N. \& Swindler, A.. (2012). "The Building Component Library: an Online Repository to Facilitate Building Energy Model Creation." In Proceedings of the 2012 ACEEE Summer Study on Energy Efficient Buildings. Pacific Grove, Calif. 
Guglielmetti, R., Macumber, D., Long, N. (2011) "OpenStudio: an open source integrated analysis platform." In Proceedings of the 12th Conference of International Building Performance Simulation Association, Sydney, Australia.

Guglielmetti, R., Scheib, J., (2012) "Challenges to Integrated Daylighting and Electric Lighting Simulation Methods in a Whole-building Energy Simulation Context." In Proceedings of the 2012 Simbuild Conference, August 2012, Madison, WI.

JSON.org. "Introducing JSON". available at http://www.json.org/.

Jiang, Y., Ming, J., Wu, D., Yen, J., Mitra, P., Messner, J.I., and Leicht, R. (2012). "BIM Server Requirements to Support the Energy Efficient Building Lifecycle", In Proceedings of the 2012 ASCE International Conference on Computing in Civil Engineering. Clearwater Beach, FL.

Jiang, Y., Ming, J., Wu, D., DeGraw, J., Lee, S., Jallow, A., Yen, J., Mitra, P., and Messner, J.I. (2013). "BIM Enabled Energy Efficient Building Analysis: Improving Software Interoperability in the AEC Community." Manuscript in preparation.

Jones, B., Bogus, S.M. (2010). "Decision Process for Energy Efficient Building Retrofits: The Owner's Perspective." August, Vol. 5, No. 3, pp. 131 - 146.

Kangaraj, G., Mahalingam, A. (2011). "Designing energy efficient commercial buildings - A systems framework. Energy Buildings." 43:2329-2343.

Klotz, L. (2011). "Cognitive biases in energy decisions during planning, design and construction of commercial buildings in the United States: An analytical framework and research needs." Energy Efficiency. 4:271-284.

O’Donnell, J., See, R., Rose, C., Maile, T., Bazjanac, V., Haves, P., (2011) "SimModel: A domain data model for whole building energy simulation.", In: IBPSA Building Simulation. Sydney, Australia.

Pollock, M., Roderick, Y., McEwan, D. \& Wheatley, C. (2009). "Building simulation as an assisting tool in designing an energy efficient building: A case study." In proceedings of 11th international conference of building simulation Glasgow. pp.1191-1198.

Walton, G., Dols, W. (2010) "CONTAM User Guide and Program Documentation." http://www.bfrl.nist.gov/IAQanalysis/docs/CWHelp30.pdf, Last updated Dec. 25th, 2010.

Weaver, E., Long N., Fleming, K., Schott, M., Benne, K. and Hale, E. (2012) "Rapid Application Development with OpenStudio." 2012 ACEEE Summer Study, Pacific Grove, California. 